\title{
Oxidized Graphene in lonic Liquids for Assembling Chemically Modified Electrodes: A Structural and Electrochemical Characterization Study
}

\author{
F. Valentini, ${ }^{\dagger}$ D. Roscioli, ${ }^{\dagger}$ M. Carbone, ${ }^{*}{ }^{\dagger}$ V. Conte, ${ }^{\dagger}$ B. Floris, ${ }^{\dagger}$ G. Palleschi, ${ }^{\dagger}$ R. Flammini, ${ }^{\ddagger}$ E. M. Bauer, ${ }^{\S}$ \\ G. Nasillo," and E. Caponetti" \\ ${ }^{\dagger}$ Department Chemical Science and Technologies, University Tor Vergata, Via della Ricerca Scientifica,1 00133 Roma \\ ${ }^{\ddagger}$ CNR-IMIP, Istituto di Metodologie Inorganiche e Plasmi, Area della Ricerca di Roma I, via Salaria km 29.300, Monterotondo Scalo \\ (RM), Italy and INFN-LNF, Laboratori Nazionali di Frascati, Via E. Fermi, 40, 00044 Frascati (RM) Italy \\ ${ }^{\S}$ Institute of Structure of Matter, Italian National Research Council, Via Salaria km 29.3, 00015 Monterotondo (RM), Italy \\ "Centro Grandi Apparecchiature, University of Palermo, Via F. Marini 14, 90128 Palermo, Italy and Department of Physical \\ Chemistry, University of Palermo,Viale delle Scienze 90128 Palermo, Italy
}

Supporting Information

ABSTRACT: Dispersions of graphene oxide (GO) nanoribbons in ionic liquids, ILs (either 1-butyl-3-methylimidazolium chloride (BMIM-Cl-) or 1-butylpyridinium chloride (-Bupy-Cl-)) have been used to assemble modified screen printed electrodes (SPEs). The graphene oxide/ionic liquid dispersions have been morphologically and structurally characterized by the use of several techniques: X-ray photoelectron spectroscopy (XPS), Fourier transform-infrared (FT-IR) spectroscopy, high-resolution-transmission electron microscopy (HR-TEM). The assembled modified SPEs have then been challenged with various compounds and compared to several electro-active targets. In all cases high peak currents were detected, as well as significant potential shifts, especially in the detection of catecholamines and $\mathrm{NADH}$, compared with the bare SPE and the conventional electrodes, such as glassy carbon (GC) and highly oriented pyrolitic graphite (HOPG). This opens the way to the assembly of new types of sensors and biosensors. The enhanced performances observed are attributed to electrocatalytic effects related to the high electrode surface area, to oxygen-assisted electron transfer, as well as to the disordering effect of the ILs, this latter related to the favorable $\pi-\pi$ interactions with the ILs and the GO plane.

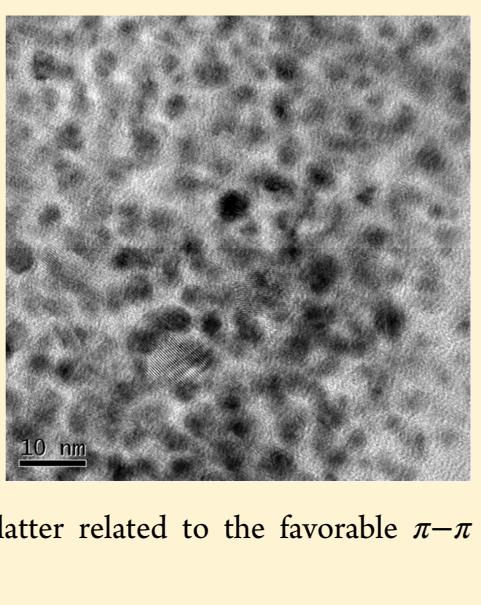

$G$ raphene is the first example of a truly two-dimensional crystal with physical and chemical properties arising from there being a gapless semiconductor where the charge carriers obey a linear dispersion relation. ${ }^{1}$ This results in a number of peculiar electronic properties, including an anomalous quantum Hall effect (QHE) and the absence of electron localization, and these suggested new perspectives for carbon-based electronics and the production of electrochemical devices. ${ }^{2}$ Several graphene preparation routes have been used: exfoliation of intercalated graphitic compounds, ${ }^{3,4} \mathrm{Si}$ sublimation from $\mathrm{SiC}$ substrates, ${ }^{5}$ micromechanical cleavage of bulk graphite, and vacuum graphitization of a single-crystal silicon carbide. ${ }^{6}$

Recently chemical exfoliation of the micrometric graphite by an oxidative chemical attack in liquid-phase yielded graphene nanoplatelets or nanoribbons. ${ }^{7}$ The same method has been applied to single-wall carbon nanotubes ${ }^{8}$ and multi-wall carbon nanotubes ${ }^{9}$ for the oxidative unzipping reaction of nanotubes, to fabricate oxidized/functionalized graphene nanoribbons. This chemical procedure also enhances the graphene thermodynamic stability, probably due to the edge termination with functional groups or chemical and physical modification of the carbon network, serving to further stabilize the graphene nanomaterials. ${ }^{10}$

The unique properties of graphene oxide GO may also play a role in electrochemistry, since the oxidation and unsaturation favor the electron transfer in detection processes. Given these circumstances, it was useful to investigate applications for GO in the fabrication of modified electrodes for the detection of several analytes of a particular bioanaytical interest. For this effort, the dispersion medium is fundamental because it determines the extent of aggregation of the GO nanoribbons and, as a consequence, the exposure of the GO to the electrochemical process.

An interesting option is offered by ionic liquids, ILs, which have been proposed as "green" alternatives to conventional solvents $^{11}$ due to their negligible vapor pressure, thermal stability, wide electrochemical potential window, low viscosity, good ionic conductivity, and recyclability. These properties

Received: May 11, 2012

Accepted: June 5, 2012

Published: June 5, 2012 
render ILs a very useful medium for liquid/liquid extraction, electrochemistry, chemical syntheses, and catalysis. ${ }^{11-13}$ The high dielectric constant of ILs provides shielding for the stacking interactions resulting from van der Waals interactions and helps to disperse these nanomaterials effectively. In particular, the distribution and the electronic and electrochemical features of the nanomaterial can be tuned (ref 14 and references therein) by regulating the water content and type of counterions present in the ILs. ${ }^{4}$

In this study, in order to investigate solvent effects on the electrochemical performance and responses of the resulting SPEs, we used GO dispersed in two ionic liquids: 1-butyl-3methylimidazolium chloride (BMIM-Cl) and 1-butylpyridinium chloride (Bupy-Cl) (sketched in Scheme 1).

Scheme 1. Molecular Structures of (a) BMIM-Cl and (b) Bupy-Cl Ionic Liquids
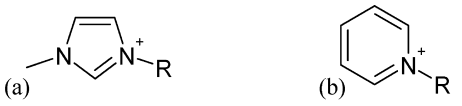

The GO were synthesized as described in our previous work, ${ }^{8,9}$ seeking conditions to obtain stable and homogeneous nanodispersions. After morphological and structural characterization, these were used to assemble (via a drop casting method) the graphene/IL based SPEs. Finally, we have performed an electrochemical study to characterize the behavior of the modified electrodes SPEs with respect to several electro-active probes, both inorganic and organic ones of biological interest. For all of them we observed an enhancement of the electron-transfer efficiency and an improvement in the electrochemical stability of the resulting $I$ vs $V$ profiles.

\section{EXPERIMENTAL SECTION}

The assembly and testing of the graphene oxide modified SPEs was the final phase of a long procedure. First, GO and ILs were synthesized and subjected to a structural characterization. Then $\mathrm{GO} / \mathrm{IL}$ dispersions were produced and the effects of the dispersion were evaluated by spectroscopic and microscopic characterization. Modified SPEs were assembled and the homogeneity of the coating was investigated by spectroscopic and electrochemical techniques. Finally, the performance of the modified SPEs were tested against several organic, inorganic, and biochemical analytes. Details on the sample preparation and materials are given in the Supporting Information.

\section{APPARATUSES AND PROCEDURES}

Structural and Morphological Characterizations. $X$-ray Photoelectron Spectroscopy (XPS). XPS measurements were made on GO powder and on the GO in dispersion and subsequent deposition (BMIM-Cl) or centrifugation (Bupy$\mathrm{Cl}$ ). The GO dispersion was deposited on a silicon wafer until complete coverage of the surface. The measurements were performed at the INFN-LNF surface laboratory of Frascati (RM), under UHV conditions (base pressure lower than $2 \times$ $10^{-10}$ mbar). The UHV system includes an XPS analysis chamber and a chamber for in situ preparation of the samples. The two experimental chambers are connected, allowing for measurements on the same sample without exposure to the atmosphere. Photoemission spectra have been acquired with an EAC125 electron analyzer. $\mathrm{Mg} \mathrm{K} \alpha$ photons $(h \nu=1253.6 \mathrm{eV})$ were used to induce electron emission. The X-ray beam intensity was set to $36 \mathrm{~W}(12 \mathrm{kV} \times 3 \mathrm{~mA})$ in order to ensure a good signal-to-noise ratio.

Fourier Transform-Infrared (FT-IR). FT-IR spectra were recorded on a Shimadzu Prestige 21 spectrophotometer in the region 400-4000 $\mathrm{cm}^{-1}$. Solid GO, BMIM, and Bupy were included in $\mathrm{KBr}$ pellets. Spectra on liquids and dispersions were taken by using liquid sample holders without any further sample treatment.

High-Resolution-Transmission Electron Microscopy (HRTEM). HR-TEM analyses were performed on GO and on the GO/ILs dispersions. A JEM-2100 (JEOL, Japan) microscope was used, operating at $200 \mathrm{kV}$ accelerating voltage and equipped with an energy dispersive $\mathrm{X}$-ray spectrometer (EDS) (Oxford Instruments, U.K.) suited for element identification. The acquired high-resolution micrographs were obtained by the inverse fast fourier transformer (IFFT), equipped with the Digital-Micrograph GATAN software. The samples were prepared by placing a drop of GO/IL dispersions on a copper grid, leaving the solvent to evaporate.

Scanning Electron Microscopy (SEM). The morphological characterization of the modified SPEs has been performed by using a field emission-scanning electron microscope/energy dispersive X-ray analyzer (FE-SEM/EDX, LEO 1550) equipped with a sputter coater (Edwards Scan Coat K550X). A volume of $6 \mu \mathrm{L}$ of the dispersions has been directly deposited (by drop coating) on the SPE surface, leaving the solvent to evaporate at room temperature (RT). The modified SPEs were fixed on the aluminum stub, with carbon tape. The samples were then coated by a thin $\mathrm{Au}$ layer (with a thickness 10-20 nm), deposited by sputtering for $2 \mathrm{~min}$ at $I=25 \mathrm{~mA}$.

Electrochemical Determinations. Cyclic Voltammetry (CV) and Chronoamperometry. Modified SPEs were assembled by drop coating $100 \mu \mathrm{L}$ of GO/IL dispersion solutions after testing several dispersion concentrations. This value guarantees the complete coverage of the three electrodebased electrochemical cell. These measurements are made in drop detection mode as presented in our previous paper ${ }^{15}$ and not in batch or flow injection analysis (FIA). In particular, GO/ IL dispersions contained $90 \mu \mathrm{L}$ of GO/ILs (where ILs/ $\mathrm{H}_{2} \mathrm{O}=$ $50: 50 \% \mathrm{w} / \mathrm{w}$ ) and $10 \mu \mathrm{L}$ of the different analyte solutions, at different concentration values: $1 \mathrm{mM}$ in the case of potassium ferricyanide, hexaammineruthenium(III) chloride, sodium hexachloroiridate-(III) hydrate, $\mathrm{NADH}$, ascorbic acid, and dopamine. A concentration of $0.1 \mathrm{mM}$ was investigated in the case of epinephrine, 3,4-dihydroxyphenylacetic acid (DOPAC), serotonin, and caffeic acid. All experiments were carried out at room temperature, and all solutions were deaerated under a nitrogen stream. Cyclic voltammetry experiments were performed at a scan rate of $100 \mathrm{mV} / \mathrm{s}$ over the relevant potential range using $0.2 \mathrm{M}$ phosphate buffer $(\mathrm{pH}$ $=7.0$ ). Only for $\mathrm{NADH}$, a scan rate of $10 \mathrm{mV} / \mathrm{s}$ was used. Furthermore for ascorbic acid, the measurements have been carried out in acetate buffer solution, at $\mathrm{pH}$ of 5.4. Chronoamperometry was performed in a $1 \mathrm{mM}$ potassium ferricyanide solution at an applied potential of $+300 \mathrm{mV}$, vs $\mathrm{Ag}$ / $\mathrm{AgCl}$, for $1 \mathrm{~min}$ in order to calculate the electrochemical area by the Cottrell approach, as reported later in the text. Experiments were performed using an Autolab electrochemical system (Eco Chemie, Utrecht, The Netherlands) equipped with PGSTAT-12 and GPES software (Eco Chemie, Utrecht, The Netherlands). 


\section{RESULTS AND DISCUSSION}

Structural and Morphological Characterization of GO and GO/IL Dispersions. GO and GO/ILs dispersions have been characterized by XPS, FT-IR, and by HR-TEM. All investigations indicate the presence of oxidized groups on the graphene nanoribbons and significant interactions between the GO and the ILs.

XPS spectra were recorded for GO as a powder and then upon dispersion in BMIM-Cl and Bupy-Cl. In the former case, water was used as the depositing agent on the supporting silicon wafer. As for GO in ILs, centrifugation was performed on the dispersions and the centrifugate was deposited on the silicon wafers. In this procedure and for both ILs, a separation of weakly bonded ILs occurs, whereas only more strongly bonded molecules would remain attached.

Broad scans up to $1100 \mathrm{eV}, \mathrm{C} 1 \mathrm{~s}, \mathrm{~N} 1 \mathrm{~s}$, and O1s spectra were taken for all the samples. The C1s and O1s spectra of GO, when deposited from water or from the Ils, are nearly indistinguishable. In Figure 1 we report the GO-BMIM-Cl

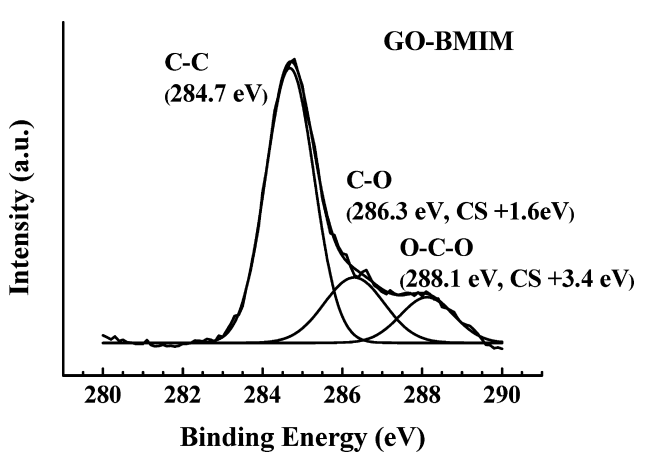

Figure 1. C1s spectrum of GO dispersed in BMIM-Cl, centrifuged, and deposited on a silicon wafer.

C1s spectrum. It can be deconvoluted into three peaks, a main peak at $284.7 \mathrm{eV}$, one at $286.3 \mathrm{eV}$ (chemical shift, CS, $+1.6 \mathrm{eV}$ ) and a third one at $288.1 \mathrm{eV}(\mathrm{CS}+3.4 \mathrm{eV})$. These values are similar to what was reported by Stankovich et al. ${ }^{16}$ who synthesized and characterized GO by graphite oxide exfoliation. They attributed the main peak to aromatic carbon, and the higher energy peaks $(\mathrm{CS}+1.6$ and $+3.4 \mathrm{eV})$ to $\mathrm{C}-\mathrm{O}$ and $\mathrm{C}=$ $\mathrm{O}$, respectively. They also found another small peak at $288.9 \mathrm{eV}$
(CS +4.2 eV), which can be attributed to carbon from $\mathrm{C}(\mathrm{O}) \mathrm{OH}$.

In our case, we did not find the highest energy peak of the carboxylate in the C1s spectrum. We also do not observe the stretching vibrations of carbonyl $\mathrm{C}=\mathrm{O}$ groups and carboxylic acid $\mathrm{C}(\mathrm{O}) \mathrm{OH}$ groups in the infrared spectra (next section). As a consequence, for our samples we must attribute the peak at $\mathrm{CS}+3.4 \mathrm{eV}$ to carbon bonded twice to hydroxylic groups. Neither did we observe a C1s component at CS $+1.2 \mathrm{eV}$, which can be attributed to carbon bonded to nitrogen $(\mathrm{C}-\mathrm{N})$, nor do we find any trace of nitrogen in any of the wide scan or N1s spectra of all the samples. Hence, we rule out the occurrence of any strong interaction between GO and any of the ILs. Weak interactions, however, do occur and their effect can be appreciated both by infrared and by HR-TEM.

Figure 2a,b reports the FT-IR spectra of GO as a powder and upon dispersion in BMIM-Cl solutions and in Bupy-Cl, respectively. Furthermore, an IR spectrum has been taken on GO deposited from the BMIM-Cl dispersion after $2-3$ days. The same could not be performed on GO/Bupy-Cl, since this is a much more stable dispersion and no deposit was observed after months. IL solution spectra were also taken and are included in the corresponding figures.

Peaks for the GO are found at $620,1114 \mathrm{~cm}^{-1}$ with two shoulders at 1147 and $1165 \mathrm{~cm}^{-1}$, at $1385,1633 \mathrm{~cm}^{-1}$ and a broad band centered at approximately $3400 \mathrm{~cm}^{-1}$. The first band is assigned to the aromatic skeletal mode while the whole set of bands and shoulders around $1165 \mathrm{~cm}^{-}$is due to the $\mathrm{C}-\mathrm{O}$ stretching vibration of an alkoxy group. The bands at 1385 and $3400 \mathrm{~cm}^{-1}$ are typical for the presence of hydroxyl groups and are assigned to $\mathrm{O}-\mathrm{H}$ deformation and stretching vibrations respectively. A lack of bands around $1730 \mathrm{~cm}^{-1}(-\mathrm{C}=\mathrm{O}$ stretching $)^{17-19}$ excludes the presence of carbonyl and/or carboxyl groups, in agreement with the XPS findings.

Both BMIM-Cl and Bupy-Cl solutions show very similar spectra. This similarity is not surprising, since the few observable bands at 645,1620 , and $3400 \mathrm{~cm}^{-1}$ are characteristic of water and not really ascribable to the ionic liquids (the band at $2100 \mathrm{~cm}^{-1}$ can be attributed to an impurity). However, it is important to note that the dispersion of GO in either BMIM-Cl or Bupy- $\mathrm{Cl}$ does not change the overall infrared spectra, which still look like of the water-alone type, in spite the presence of the otherwise sharp-peaked GO. The quenching of the GO vibration peaks can be related, among the others, to $\pi-\pi$
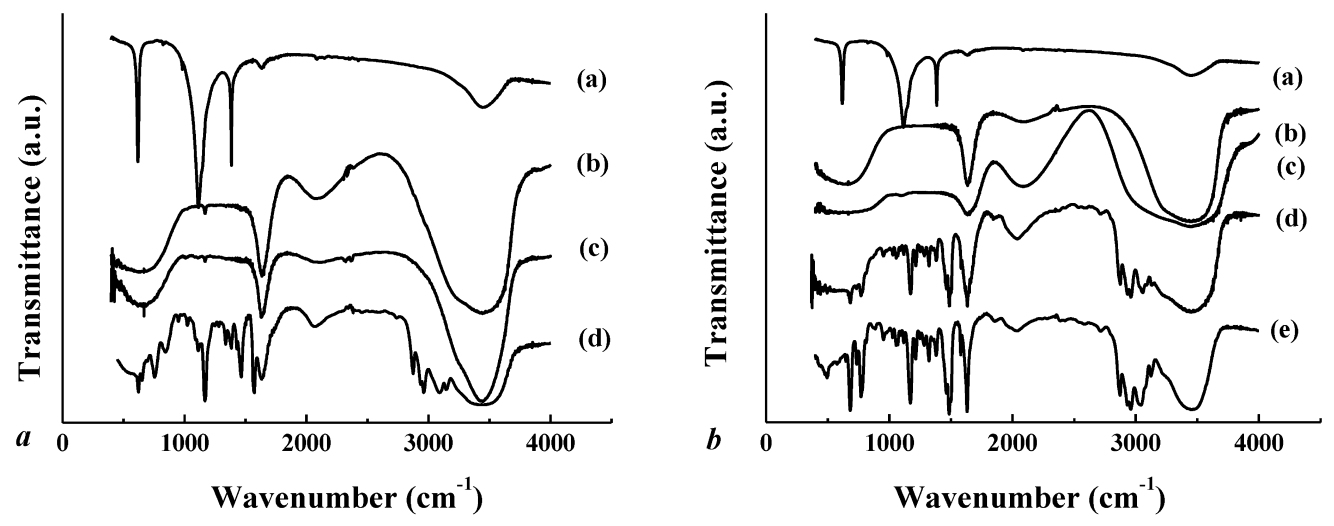

Figure 2. (a) Infrared spectra of (a) graphene oxide (GO), (b) BMIM-Cl, (c) GO dispersed in BMIM-Cl, and (d) GO upon spontaneous deposition from BMIM-Cl. (b) Infrared spectra of (a) graphene oxide (GO), (b) Bupy-Cl, (c) GO dispersed in Bupy-Cl, (d) GO centrifuged from a GO/Bupy$\mathrm{Cl}$ dispersion, and (e) pure Bupy-Cl. 
interactions, which may occur between the aromatic systems of the graphene oxide and ionic liquids. Traces of such an interaction can be seen in GO deposited from the BMIM-Cl dispersion, whose complex spectrum with several sharp bands can actually be completely assigned to the ionic liquid ${ }^{20}$ overlapped with $\mathrm{GO}$, in addition to the usual traces of water. As for the $\mathrm{GO} / \mathrm{Bupy}-\mathrm{Cl}$ dispersion, since no deposition of the GO occurred, the dispersion (actually more similar to a real solution) had to be centrifuged, in order to obtain a deposit. Also in this case, the infrared spectrum reveals the residual interactions between $\mathrm{GO}$ and Bupy-Cl (Figure 2b-(d)). However, since no IR spectrum was available of pure Bupy, we have recorded one here (Figure $2 b-(e)$ ) and one can notice how the two spectra can almost be overlapped.

The interactions between GO and ILs, though weak, nevertheless cause the disordering of the GO sheets. This can be seen by HR-TEM, in Figure 3a,b, where GO sheets appear well dispersed and separated due to the $\pi-\pi$ stacking interactions between the aromatic rings of the outer GO layers and the imidazolium
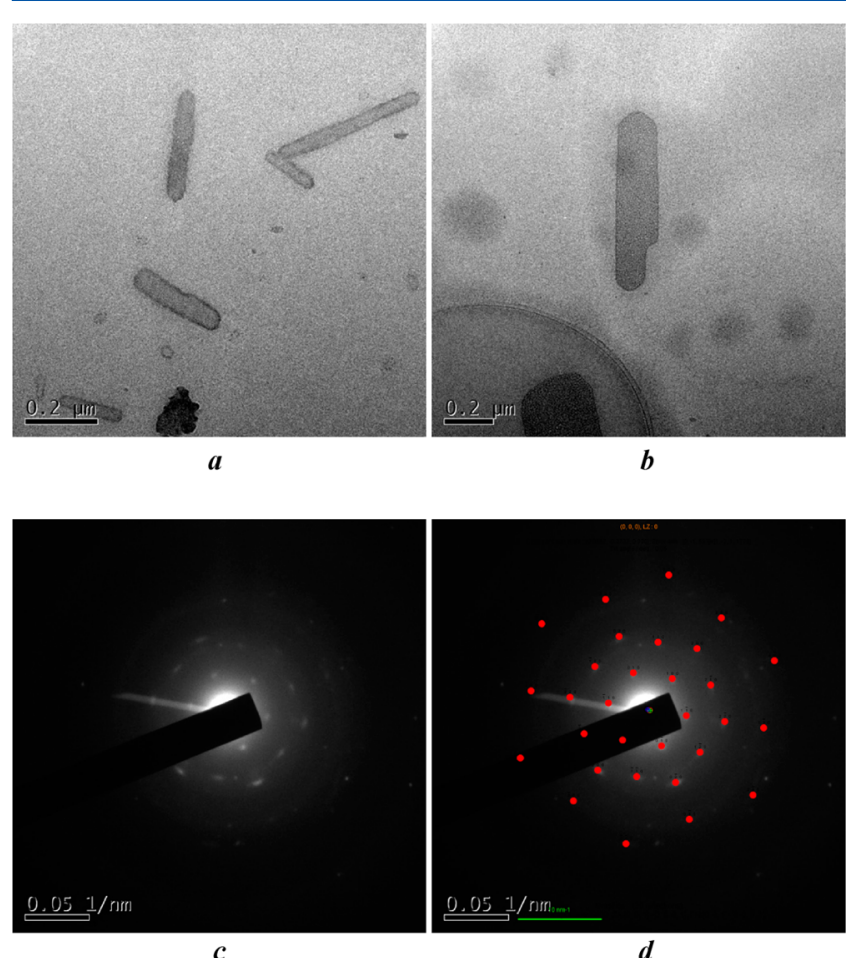

$c$

Figure 3. $(a, b)$ HR-TEM micrographs of GO nanoribbons in BMIM$\mathrm{Cl}$ dispersion $(250.000 \times)$. (c) SAED pattern of the area in the circle in parts $b$ and $d$ indexing of the diffraction spots by JEMS.

Further information can be obtained by selected area electron diffraction (SAED) patterns of GO samples. In Figure $3 c$ is reported, as an example, the SAED patterns recorded in the area indicated by the circle of Figure $3 \mathrm{~b}$. The ordered diffraction spots of Figure $3 c$ have been indexed by JEMS software $^{20}$ and the results are shown in Figure 3d. The indexing process leads to the conclusion that GO sample is composed mainly by single-layer carbon sheets with a hexagonal crystalline lattice. Many of them are oriented with the zone axis $\left[\begin{array}{lll}0 & 0 & 1\end{array}\right]$ along the optical axis.

Modified SPEs: Morphological and Electrochemical Characterization. The electrodes modified with GO/BMIM-
$\mathrm{Cl}$ and $\mathrm{GO} / \mathrm{Bupy}-\mathrm{Cl}$ dispersions were subject to SEM investigations in order to optimize the concentrations. Tests were made in the concentration range $1-20 \mathrm{mg} / \mathrm{mL}$, in steps of 2 units. The best outcome was obtained with dispersions of $\mathrm{GO} / \mathrm{ILs}$ of $1 \mathrm{mg} / \mathrm{mL}$. For all the other concentrations, macroscopic deposits on the SPE surfaces were locally observed.

Pictures of SE micrographs of the surface of the bare SPE and of the electrode modified with GO/BMIM-Cl and GO/ Bupy- $\mathrm{Cl}$ dispersions are reported in Figures $\mathrm{Sla}-\mathrm{c}$ in the Supporting Information. They exhibit a homogeneous and uniform coating, with a significant increase of the surface roughness upon modification with the dispersions.

The electrochemical characterization of our targets was performed by $\mathrm{CV}$ and was aimed at evaluating the stability of the electro-analytical signal and the electrode surface roughness, which is an expression of the electro-active area, according to the Cotrell equation ${ }^{21}$ and directly related to the peak current according to Randelss-Sevick. ${ }^{21}$

The electrochemically effective area of SPE and GN-SPEs was evaluated using chronoamperometry in a $1 \mathrm{mM}$ of potassium ferrycianide solution. The slope of the linear region of the $I-t^{-1 / 2}$ plot (not shown) in a short time region provides the product $n F A C^{0} D^{1 / 2} \pi^{-1 / 2}$ according to the Cottrell ${ }^{21}$ equation, where $C^{0}=1 \mathrm{mM}$ concentration of $\mathrm{Fe}(\mathrm{CN})_{6}^{3-}$ and $D=7.6 \times 10^{-6} \mathrm{~cm}^{2} \mathrm{~s}^{-1}, 15$ the corresponding diffusion coefficient. The other parameters have their usual meanings. ${ }^{21}$ The estimated areas calculated with the Cotrell eaquation are reported in Table 1, by chronoamperometry.

Table 1. Electrochemical Areas Estimated by the Cottrell Equation

\begin{tabular}{lc}
\multicolumn{1}{c}{ SPEs } & electrochemical area $\left(\mathrm{cm}^{2}\right)$ \\
bare & $0.01 \pm 0.0002$ \\
GO/BMIM-Cl & $0.08 \pm 0.0033$ \\
GO/Bupy-Cl & $0.12 \pm 0.0040$
\end{tabular}

CV studies are reported in Figures 4-6. A stable electroanalytical signal and good electroanalytical parameters are observed. In particular, for $\mathrm{GO} / \mathrm{Bupy}-\mathrm{Cl}$, a stable Nernstian reversible electro-analytical signal is obtained for $\mathrm{K}_{3} \mathrm{Fe}(\mathrm{CN})_{6}$ in terms of shape of the signal and quantitative electrochemical parameters. Glassy carbon (CG) and highly oriented pyrolitic graphite (HOPG) SPE electrodes were built for comparison purposes and probed for some of the targets (Figures S5 and S6 in the Supporting Information). In all cases, the GO/ILs SPEs showed better performances with respect to conventional ones, in terms of current peak intensity, potential shift, and $I$ vs $V$ profiles.

Optimization Study of the Quantitative Electrochemical Parameters. An associated scan rate study has been performed (Figures S2 and S3 in the Supporting Information). The outcome demonstrates that the electron transfer mechanism is different for the two dispersions. An adsorption process prevails during the electron-transfer reaction mechanism, hinting that a fouling effect might occur at the GO/BMIM-Cl modified SPEs. A diffusion-controlled process occurs, instead, in the case of GO/Bupy-Cl.

Although the wave shapes displayed by the two electrode types in Figures 4-6 and Figures S2 and S3 in the Supporting Information are rather similar and are characteristic of a diffusion-controlled response, an adsorption process occurs at 

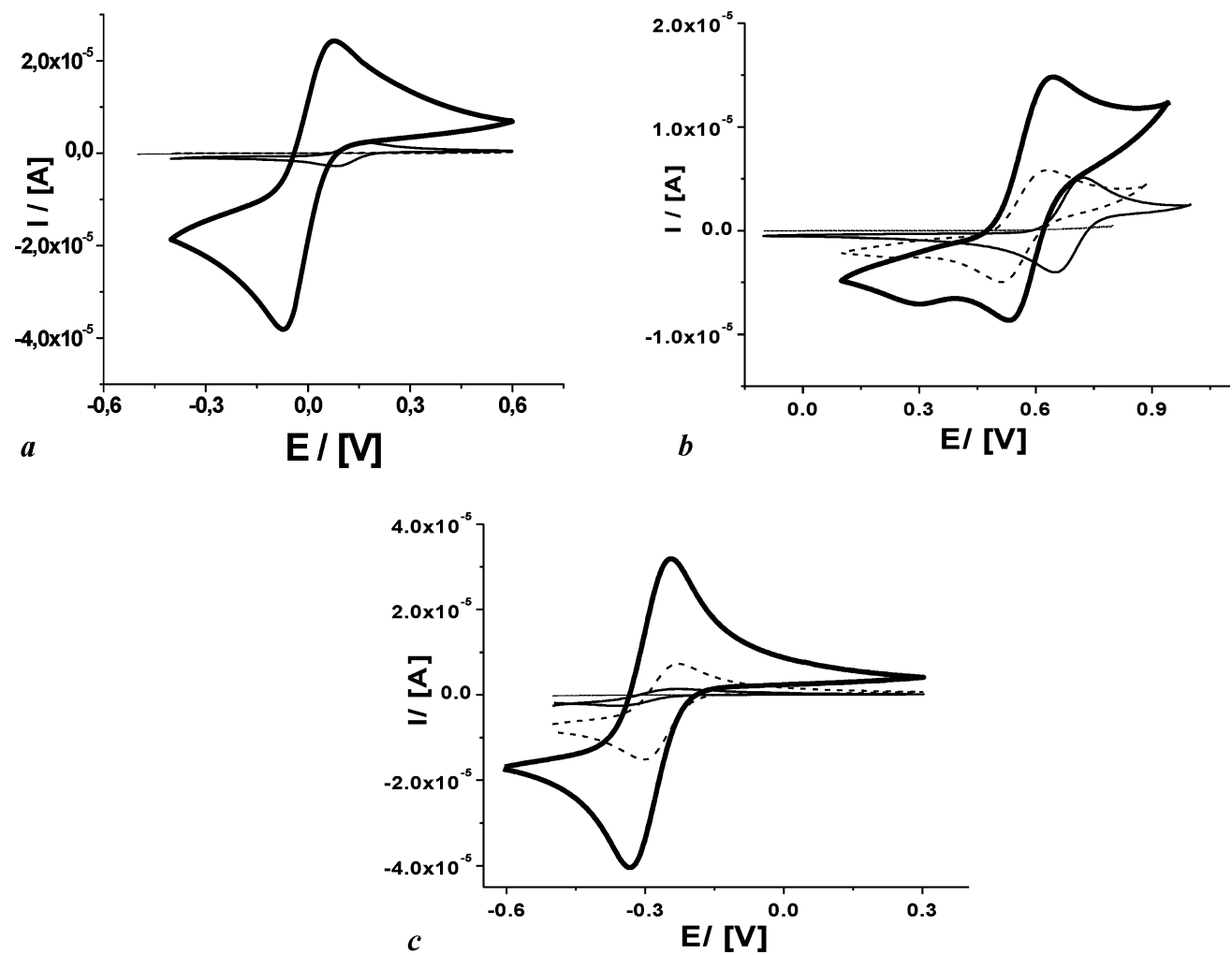

Figure 4. Cyclic voltammograms of (a) $1 \times 10^{-3} \mathrm{M} \mathrm{Fe}(\mathrm{CN})_{6}{ }^{3-}$; (b) $1 \times 10^{-3} \mathrm{M} \mathrm{Na}_{3}\left(\mathrm{IrCl}_{6}\right) \cdot \mathrm{H}_{2} \mathrm{O}$; and (c) $1 \times 10^{-3} \mathrm{M} \mathrm{Ru}\left(\mathrm{NH}_{3}\right)_{6}{ }^{3+}$. Experimental

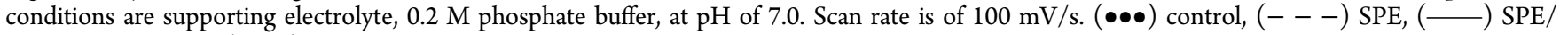
$\mathrm{GO} / \mathrm{BMIM}-\mathrm{Cl}$, and ( $\stackrel{-}{\longrightarrow} \mathrm{SPE} / \mathrm{GO} / \mathrm{Bupy}-\mathrm{Cl}$.

the SPE modified by using GO/BMIM-Cl at the second voltammetric cycle, and this effect was not observed in the case of the modified SPE by using GO/Bupy-Cl. This is probably related to the weaker $\pi-\pi$ interactions in the case of $\mathrm{GO} /$ BMIM-Cl modified SPEs, with respct to GO/Bupy-Cl. This is also supported by the spontaneous deposition occurred from the BMIM-Cl dispersion after 2-3 days, at variance with the stable Bupy-Cl dispersion (actually closer to a solution, than to a dispersion).

Taking account of the signal-to-noise ratio and the current peak values (Table 2), the scan rate value of $100 \mathrm{mV} / \mathrm{s}$ has been selected to perform all the subsequent measurements. $i_{\mathrm{pa}}$ and $i_{\mathrm{pc}}$ in the table and graphs are the intensity of the anodic and cathodic peak current, respectively.

Regarding possible chloride related potential shifts, when working in drop mode, we can exclude them because chloride was the counteranion of ionic liquids composition, and its concentration is constant, during measurements. We performed comparison CVs at scan rate of $100 \mathrm{mV} / \mathrm{s}$, in $1 \times 10^{-3} \mathrm{M}$ of $\mathrm{Fe}(\mathrm{CN})_{6}^{3-}$ solution in (a) drop mode, working with a pseudoreference electrode, directly inserted in the SPE configuration, (b) batch mode as well as with (c) GC conventional electrode $(\phi=3 \mathrm{~mm})$ with a secondary reference electrode, $\mathrm{Ag} / \mathrm{AgCl} / \mathrm{Cl}^{-}$and a Pt counter (auxiliary) electrode, working in a three compartment electrolysis cell. The $3 \mathrm{CV}$ profiles show negligible shifts (Figure S4 in the Supporting Information).

Electrochemical Studies. Several molecules (inorganic, organic, and of biological interest) have been tested, in terms of their electrochemical detection. In particular, we probed potassium ferrycianide, iridium chloride, hexaammineruthenium(III) chloride, among the inorganic compounds; ascorbic and caffeic acids among the organic ones, and the neurotransmitters dopamine (DOPA), epinephrine, nor-epinephrine, and serotonin. Furthermore, we also tested the performance of the electrodes with respect to $\mathrm{NADH}$. The specific biomolecules were chosen because their determination can be useful in a wide range of applications; among them, the construction of detection devices for neurotransmitters in cases of Parkinson's and Alzheimer's disorders. Quantitative assessment of NADH can be used in assembling dehydrogenase-based biosensors and ethanol fuel cells. $^{22}$ Finally, the determination of caffeic acid is important in the development of chemical sensors for alkaloid detection in food matrixes. ${ }^{23}$ Detection of all these molecules has been fully characterized from an electrochemical point of view, and all electro-analytical parameters have been reported in Table 2 . CVs for inorganic, organic, and biomolecules are reported in Figures 4, 5, and 6, respectively.

For all the molecules, we observe a peak current ratio $\sim 1$ (for the reversible targets) or an increased current value (for the quasi-reversible and irreversible ones) for both types of modified SPEs with respect to the bare one and especially compared with the conventional electrode, as GC and HOPG. This is most likely related to the higher electrochemically active area, strictly related to the surface nominal area of GO (evaluated in this work by the BET approach, resulting in 1200 $\mathrm{m}^{2} / \mathrm{g}$, data not shown) and also to the roughness of the modified SPE surfaces. In particular, in the case of $\mathrm{NADH}$, GO/Bupy-Cl exhibited a better electrochemical signal, both in terms of stability and higher current peak values. Furthermore, the NADH voltammograms show a reduced fouling upon further cycle testing. The peak intensity of the first cycle is reduced by $50 \%$ at the second cycle and remains constant 
Table 2. Electrochemical Parameters of the Electro-Active Targets, Collected at GO/BMIM-Cl and GO/Bupy-Cl Modified, Bare SPE, CG and HOPG ${ }^{a}$

\begin{tabular}{|c|c|c|c|c|c|}
\hline \multicolumn{3}{|c|}{$i_{\mathrm{pa}} ; i_{\mathrm{pc}}(\mu \mathrm{A})$} & \multicolumn{3}{|c|}{$\Delta E_{\mathrm{p}} ; E_{\mathrm{p} a} j_{\mathrm{pc}}(\mathrm{mV})$} \\
\hline target & $\begin{array}{l}\text { SPE }(\phi \\
=3 \mathrm{~mm})\end{array}$ & $\begin{array}{c}\text { SPE/ } \\
\text { GO/ } \\
\text { BMIM-Cl }\end{array}$ & $\begin{array}{c}\text { SPE/ } \\
\text { GO/ } \\
\text { Bupy-Cl }\end{array}$ & $\begin{array}{c}\mathrm{GC}(\phi \\
=3 \\
\mathrm{~mm})\end{array}$ & $\begin{array}{l}\mathrm{HOPG}(\phi \\
=3 \mathrm{~mm})\end{array}$ \\
\hline $\begin{array}{l}\mathrm{Fe}(\mathrm{CN})_{6}^{3-} 1 \\
\mathrm{mM}\end{array}$ & $\begin{array}{c}i_{\mathrm{pa}} / i_{\mathrm{pc}}= \\
1.0 \\
\Delta E_{\mathrm{p}}= \\
390\end{array}$ & $\begin{array}{c}i_{\mathrm{pa}} / i_{\mathrm{pc}}= \\
\quad 1.0 \\
\Delta E_{\mathrm{p}}=77\end{array}$ & $\begin{array}{c}i_{\mathrm{pa}} / i_{\mathrm{pc}}= \\
0.9 \\
\Delta E_{\mathrm{p}}= \\
132\end{array}$ & $\begin{array}{c}i_{\mathrm{pa}} / i_{\mathrm{pc}}= \\
0.4 \\
\Delta E_{\mathrm{p}}= \\
268\end{array}$ & n.d. \\
\hline $\begin{array}{l}\mathrm{Na}_{3} \mathrm{IrCl}_{6} \cdot \mathrm{H}_{2} \mathrm{O} \quad 1 \\
\mathrm{mM}\end{array}$ & $\begin{array}{c}i_{\mathrm{pa}} / i_{\mathrm{pc}}= \\
0.9 \\
\Delta E_{\mathrm{p}}= \\
110\end{array}$ & $\begin{array}{l}i_{\mathrm{pa}} / i_{\mathrm{pc}}= \\
1.2 \\
\Delta E_{\mathrm{p}}=69\end{array}$ & $\begin{array}{c}i_{\mathrm{pa}} / i_{\mathrm{pc}}= \\
1.3 \\
\Delta E_{\mathrm{p}}=88\end{array}$ & n.d. & n.d. \\
\hline$\underset{\mathrm{mM}}{\mathrm{Ru}\left(\mathrm{NH}_{3}\right)_{6}{ }^{3+} 1}$ & $\begin{array}{c}i_{\mathrm{pa}} / i_{\mathrm{pc}}= \\
1.1 \\
\Delta E_{\mathrm{p}}= \\
66\end{array}$ & $\begin{array}{c}i_{\mathrm{pa}} / i_{\mathrm{pc}}= \\
1.0 \\
\Delta E_{\mathrm{p}}= \\
110\end{array}$ & $\begin{array}{c}i_{\mathrm{pa}} / i_{\mathrm{pc}}= \\
1.1 \\
\Delta E_{\mathrm{p}}=83\end{array}$ & $\begin{array}{c}i_{\mathrm{pa}} / i_{\mathrm{pc}}= \\
1.7 \\
\Delta E_{\mathrm{p}}= \\
94\end{array}$ & n.d. \\
\hline $\begin{array}{l}\text { caffeic acid } 1 \\
\text { mM }\end{array}$ & $\begin{array}{c}i_{\mathrm{pa}} / i_{\mathrm{pc}}= \\
1.2 \\
\Delta E_{\mathrm{p}}= \\
406\end{array}$ & $\begin{array}{c}i_{\mathrm{pa}} / i_{\mathrm{pc}}= \\
1.4 \\
\Delta E_{\mathrm{p}}= \\
434\end{array}$ & $\begin{array}{c}i_{\mathrm{pa}} / i_{\mathrm{pc}}= \\
1.3 \\
\Delta E_{\mathrm{p}}= \\
412\end{array}$ & n.d. & n.d. \\
\hline $\begin{array}{l}\text { ascorbic acid } 0.1 \\
\mathrm{mM}\end{array}$ & $\begin{array}{c}i_{\mathrm{pa}}=3.0 \\
E_{\mathrm{pa}}= \\
166\end{array}$ & $\begin{array}{l}i_{\mathrm{pa}}=1.5 \\
E_{\mathrm{pa}}=362\end{array}$ & $\begin{array}{l}i_{\mathrm{pa}}=9.0 \\
E_{\mathrm{pa}}=300\end{array}$ & $\begin{array}{c}i_{\mathrm{pa}}=1.0 \\
E_{\mathrm{pa}}= \\
388\end{array}$ & n.d. \\
\hline & $\begin{array}{l}i_{\mathrm{pa}}=\text { n.d. } \\
E_{\mathrm{pa}}= \\
\text { n.d. }\end{array}$ & $\begin{array}{l}i_{\mathrm{pa}}=7 \\
E_{\mathrm{p}}=500\end{array}$ & $\begin{array}{l}i_{\mathrm{pa}}=50 \\
i_{\mathrm{pc}}=-15\end{array}$ & $\begin{array}{l}i_{\mathrm{pa}}=13 \\
i_{\mathrm{pc}}=-4\end{array}$ & $\begin{array}{l}i_{\mathrm{pa}}=4.5 \\
i_{\mathrm{pc}}=-5\end{array}$ \\
\hline $\begin{array}{l}\text { dopamine } 0.1 \\
\text { mM }\end{array}$ & & & $E_{\mathrm{pa}}=300$ & $E_{\mathrm{pa}}=$ & $\Delta E_{\mathrm{p}}=141$ \\
\hline & & & $\begin{array}{l}E_{\mathrm{pc}}= \\
\quad-150\end{array}$ & $E_{\mathrm{pc}}=74$ & \\
\hline epinephrine 0.1 & $\begin{array}{c}i_{\mathrm{pa}}=3.9 \\
E_{\mathrm{pa}}= \\
279\end{array}$ & $\begin{array}{l}i_{\mathrm{pa}}=14 \\
i_{\mathrm{pc}}=-6.0\end{array}$ & $\begin{array}{c}i_{\mathrm{pa}}=11 \\
i_{\mathrm{pc}}= \\
-4.2\end{array}$ & $\begin{array}{c}i_{\mathrm{pa}}=3.5 \\
E_{\mathrm{pa}}= \\
492\end{array}$ & $\begin{array}{l}i_{\mathrm{pa}}=2.0 \\
E_{\mathrm{pa}}=550\end{array}$ \\
\hline $\mathrm{mM}$ & & $\begin{array}{l}E_{\mathrm{pa}}=330 \\
E_{\mathrm{pc}}= \\
\quad-300\end{array}$ & $\begin{array}{c}E_{\mathrm{pa}}=279 \\
E_{\mathrm{pc}}= \\
\quad-325\end{array}$ & & \\
\hline & $\begin{array}{c}i_{\mathrm{pa}}=3.0 \\
i_{\mathrm{pc}}= \\
-2.9\end{array}$ & $\begin{array}{l}i_{\mathrm{pa}}=10.0 \\
i_{\mathrm{pc}}=-8.5\end{array}$ & $\begin{array}{l}i_{\mathrm{pa}}=3.5 \\
i_{\mathrm{pc}}= \\
\quad-3.0\end{array}$ & $\begin{array}{l}i_{\mathrm{pa}}=2.5 \\
E_{\mathrm{pa}}= \\
471\end{array}$ & $\begin{array}{l}i_{\mathrm{pa}}=2.0 \\
E_{\mathrm{pa}}=466\end{array}$ \\
\hline mM & $\begin{array}{l}E_{\mathrm{pa}}= \\
200\end{array}$ & $E_{\mathrm{pa}}=396$ & $E_{\mathrm{pa}}=380$ & & \\
\hline & $\begin{array}{l}E_{\mathrm{pc}}= \\
\quad-350\end{array}$ & $\begin{array}{l}E_{\mathrm{pc}}= \\
\quad-100\end{array}$ & $\begin{array}{l}E_{\mathrm{pc}}= \\
\quad-180\end{array}$ & & \\
\hline $\begin{array}{l}\text { serotonine } 0.1 \\
\mathrm{mM}\end{array}$ & $\begin{array}{c}i_{\mathrm{pa}}=2.8 \\
E_{\mathrm{pa}}= \\
197\end{array}$ & $\begin{array}{l}i_{\mathrm{pa}}=5.4 \\
E_{\mathrm{pa}}=354\end{array}$ & $\begin{array}{l}i_{\mathrm{pa}}=4.4 \\
E_{\mathrm{pa}}=311\end{array}$ & $\begin{array}{l}i_{\mathrm{pa}}=1.7 \\
E_{\mathrm{pa}}= \\
451\end{array}$ & $\begin{array}{l}i_{\mathrm{pa}}=2.1 \\
E_{\mathrm{pa}}=410\end{array}$ \\
\hline $\mathrm{NADH} 0.1 \mathrm{mM}$ & $\begin{array}{c}i_{\mathrm{pa}}=2.4 \\
E_{\mathrm{pa}}= \\
344\end{array}$ & $\begin{array}{l}i_{\mathrm{pa}}=1.6 \\
E_{\mathrm{pa}}=581\end{array}$ & $\begin{array}{l}i_{\mathrm{pa}}=3.7 \\
E_{\mathrm{pa}}=570\end{array}$ & $\begin{array}{l}i_{\mathrm{pa}}=2.3 \\
E_{\mathrm{pa}}= \\
\quad 700\end{array}$ & $\begin{array}{l}i_{\mathrm{pa}}=2.5 \\
E_{\mathrm{pa}}=810\end{array}$ \\
\hline
\end{tabular}

${ }^{a}$ For the eversible targets, current peak ratio and peak-to-peak potential separation are reported. For the quasi-reversible ones, anodic and cathodic current and potential peaks are reported. For the irreversible targets, anodic current and anodic potential peaks are reported.

during the third cycle. This is remarkable for a $\mathrm{NADH}$ detection, since typically only one cycle can be performed without passivation with conventional electrodes. ${ }^{24}$ Also the oxidation potentials are significantly lower than with GC and HOPG electrodes. So, we built and tested GC and HOPG electrodes and compared the performances of these vs GO/ILs electrodes. The corresponding voltammograms are reported in Figures S5 and S6 in the Supporting Information, and the main electrochemical parameters in Table 2.
A comparison of $\mathrm{CVs}$ for $\mathrm{NADH}$ performed with electrodes with $\mathrm{GO} / \mathrm{Bupy}-\mathrm{Cl}, \mathrm{GO} / \mathrm{BMIM}-\mathrm{Cl}$, the ionic liquids alone as well as GO alone (Figure S7 in the Supporting Information) clearly show the better performances of the GO/ILs dispersions, indicating a synergistic electro-catalytic effect of GO and ionic liquids. The synergy may be explained by the capability of ILs to form an electron-conducting as well as an ion-conducting network, which may improve electronic and ionic transport capacity and allow the electro-oxidation of $\mathrm{NADH}$ at a lower potential. The favorable electrochemical $\mathrm{NADH}$ oxidation might be related to the charge-charge interactions between the $\mathrm{NADH}$ and the positively charged imidazolium (BMIM) and pyridinium (Bupy) ions. This hypothesis is supported by the apparent kinetic parameters, evaluated according to the Tafel equation. ${ }^{21}$ The charge transfer coefficient $(\alpha)$ and the standard rate constant $(K)$ for $\mathrm{NADH}$ oxidation at SPE/GO/bupyCl modified electrode are calculated and equal to 0.32 and $4.56 \times 10^{-8} \mathrm{~cm} \mathrm{~s}^{-1}$, respectively. The value of $\alpha$ is lower than the expected value (usually 0.5 ), which results from a dissymmetry of the potential energy curves of the reactant and products in the context of Butler-Volmer kinetics suggesting the irreversibility of the oxidation of $\mathrm{NADH} .^{25}$ At the same time, the $K$ value is almost 12 times as large as that $\left(K=3.73 \times 10^{-9} \mathrm{~cm} \mathrm{~s}^{-1}\right)$ obtained in this study, working at a GC electrode, similar to that reported in the literature. ${ }^{25}$ In the case of SPE/GO/bmimCl modified electrode, $\alpha(0.30)$ is quite similar to that calculated above for $\mathrm{SPE} / \mathrm{GO} /$ bupy, while the corresponding $K$ resulted in $8.42 \times$ $10^{-9} \mathrm{~cm} \mathrm{~s}^{-1}$.

For dopamine, GO/Bupy-Cl showed interesting results, since high current peaks were recorded and better electrochemical stability was attained. In this case, we find that there is a significant electrocatalytic effect, since compared to the bare electrode that shows only a capacitive current, the GO/Bupy-Cl electrode displays an intense anodic peak and an emerging nonnegligible cathodic wave.

In particular, the arising of the cathodic wave indicates a quasi-reversible profile, better than for GC and HOPG (Figure S8 in the Supporting Information and Table 2). The latter displays a reversible profile, though affected by a very high capacitive current in comparison with the Faradic current. GC has a quasi-reversible profile but with a peak value 1 order of magnitude lower than the GO/ILs-modified ones. Also the peak potentials of conventional electrodes are much higher than the GO/ILs modified SPEs. Furthermore, the peak-topeak separation $(\Delta E$ in the case of the quasi-reversible processes) for $\mathrm{GC}$ is also much higher than for $\mathrm{GO} / \mathrm{Bupy}-\mathrm{Cl}$ electrode (259 and $150 \mathrm{mV}$, respectively).

The charge transfer coefficient $(\alpha)$ and the standard rate constant $(K)$, calculated according to the Tafel equation ${ }^{21}$ yield the values of 0.60 for a quasi-reversible $I$ vs $V$ profile (taking into consideration that the $\alpha$ value equal to 0.5 represents the Nernstian reversible electron-transfer processes) and a $K$ value of $8.7 \times 10^{-8} \mathrm{~cm} \mathrm{~s}^{-1}$, respectively. The kinetic constant resulted in higher than expected $\left(K<1 \times 10^{-8} \mathrm{~cm} \mathrm{~s}^{-1}\right.$ for a standard GC electrode evaluated in this study and also quite similar to that described in the literature, ${ }^{26}$ for instance) and hint at an electrocatalytic effect.

The better performances of modified vs bare SPEs and the electrocatalytic effects can be related to the presence of the structural and oxidation defects in the GO nanoribbons $\left(\mathrm{sp}^{3}\right.$ hybridized carbons and edges and $-\mathrm{OH}$ groups) as well as the weak GO-ILs interactions. The former are responsible for 

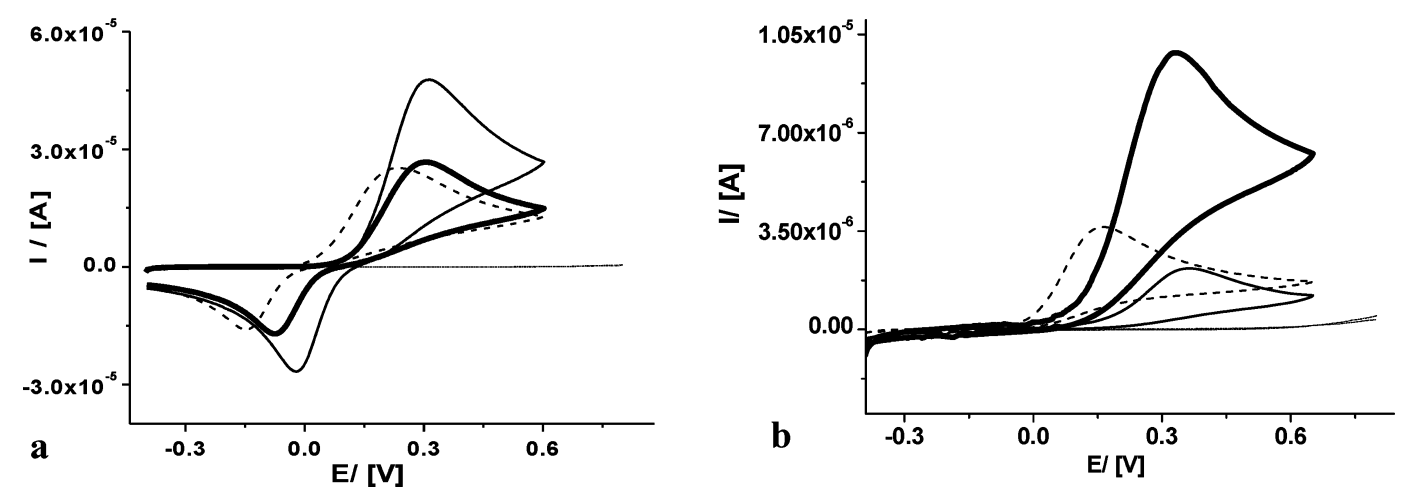

Figure 5. Cyclic voltammograms of (a) $0.1 \times 10^{-3} \mathrm{M}$ caffeic acid and (b) $1 \times 10^{-3} \mathrm{M}$ ascorbic acid. Experimental conditions for caffeic acid: supporting electrolyte, $0.2 \mathrm{M}$ phosphate buffer solution, at $\mathrm{pH}$ 7.0. Scan rate $100 \mathrm{mV} / \mathrm{s}$. For ascorbic acid: electrolyte is $0.1 \mathrm{M}$ of acetate buffer $\mathrm{pH}$

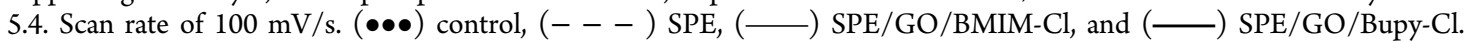
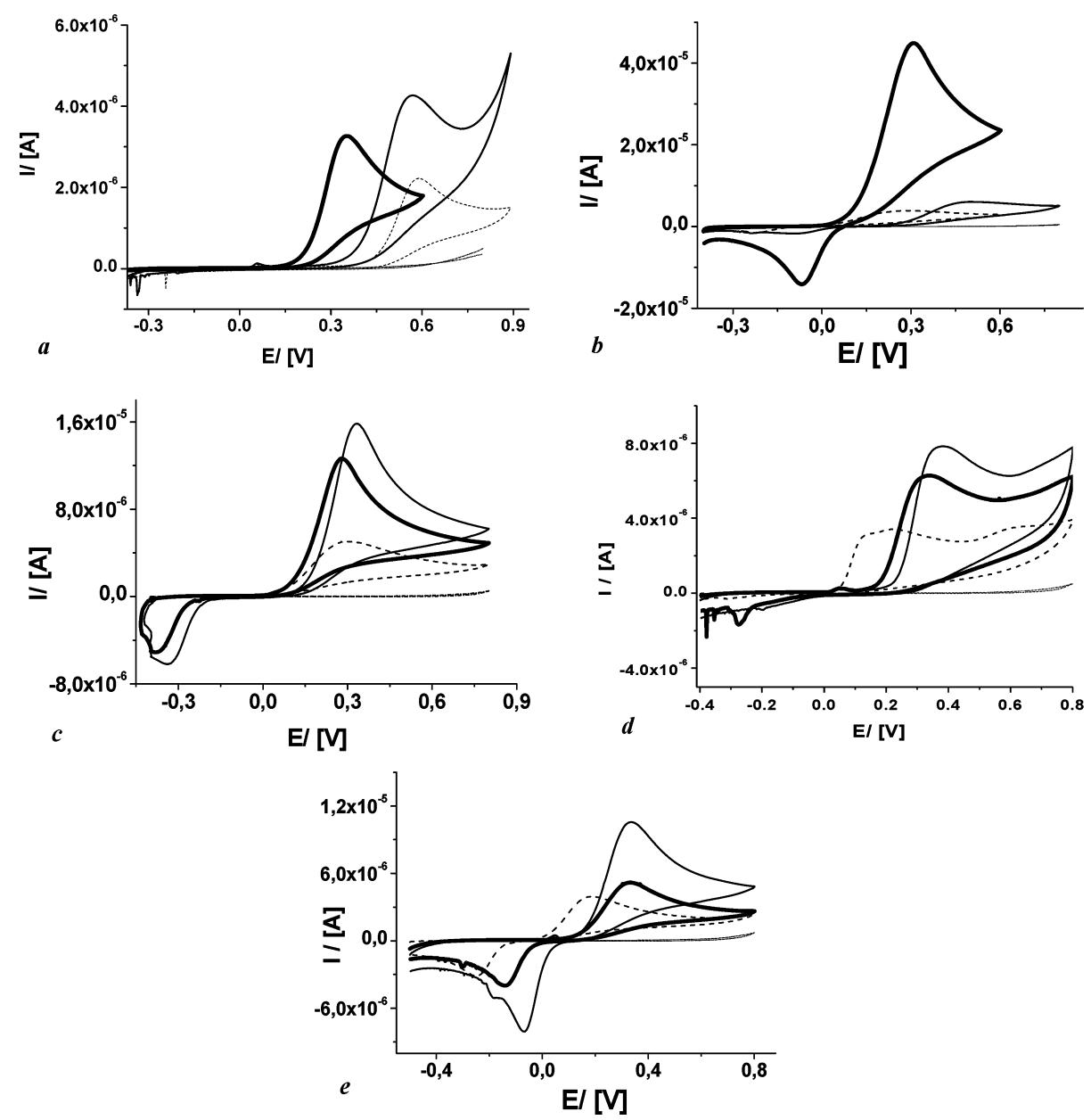

Figure 6. Cyclic voltammograms of (a) $1 \times 10^{-3} \mathrm{M} \mathrm{NADH}$, (b) $1 \times 10^{-3} \mathrm{M}$ dopamine, (c) $0.1 \times 10^{-3} \mathrm{M}$ epinephrine, (d) $0.1 \times 10^{-3} \mathrm{M}$ serotonin, and (e) $0.1 \times 10^{-3} \mathrm{M}$ DOPAC. Experimental conditions: supporting electrolyte $0.2 \mathrm{M}$ phosphate buffer solution, at $\mathrm{pH} 7.0$. Scan rate, $100 \mathrm{mV} / \mathrm{s}$,

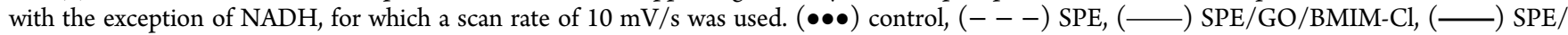
GO/Bupy-Cl.

better electron transfer based reaction mechanisms, which depends on the presence of oxidation groups. ${ }^{27} \mathrm{~A}$ weak interaction between graphene oxide and ionic liquids (as hypothesized by IR and TEM) makes the transductor surface more accessible by the analytes because several pathways are allowed for diffusion on the working electrode surface. At variance with this, a covalent solid/solvent bond increases the constraints to possible diffusion pathways and reduces the solid transport properties because of the electron localization on the bonds.

In general, it was observed that GO/Bupy-Cl modified electrodes show better performance with respect to GO/ BMIM-Cl. This may be related to the degree of interaction of Bupy-Cl and BMIM-Cl with the graphene oxide surface. Though this does not seem to affect IR and XPS significantly, the six-membered imidazolium ring of BMIM may have a better 
$\pi-\pi$ overlap with hexagonal graphene structure than the fivemembered pyridinium of Bupy, as hypothesized by Rochefort and Wuest ${ }^{28}$ in their molecular dynamics simulations and, as a consequence a slightly more efficient ordering effect, which may negatively affect the electrochemical performances, i.e., again, higher disorder yields a higher performance. The number of side chains may also play a role. This is also sketched in Figure S9 in the Supporting Information.

The oxygen containing species on the surface of the graphene sheets have a twofold effect: enhancement of the electron transfer reaction in the oxidation of catecholamines via hydride and anion-assistance of the electron transfer mechanism. ${ }^{27}$ Furthermore, the $\mathrm{sp}^{2}$ domains of the oxidized graphene nanoribbons facilitate the rapid electron-transfer and enhance the detection of several molecules. This effect was enhanced in the ionic liquids because of their disordering effect which plays a role in the dispersion of the graphene oxide, especially for Bupy- $\mathrm{Cl}$, and this may affect the accessibility of hydroxyl groups at the surface, with a resulting electro-catalytic effect.

The catalytic effects on the inorganic molecules can also be related to the presence of oxygen groups, though in the case of the enhancement of the electron transfer it may be also be explained by different and multiple reaction mechanisms, such as the formation of hydroxy- and/or aquo-hydroxy complexes (ref 27 and references cited therein).

Electron transfer kinetic constant calculated for $\mathrm{Fe}(\mathrm{CN})_{6}^{3-}$ and $\mathrm{Ru}(\mathrm{NH})_{6}{ }^{3+}$ with the GO/Bupy-Cl and GO/BMIM-Cl electrodes according to Lavagnini et al. ${ }^{29}$ that show the better performances of GO/ILs SPEs compared to standard electrodes reported in the literature (Table $1 S$ in the Supporting Information). The $\mathrm{GO} / \mathrm{Bupy}-\mathrm{Cl}$ electrochemical performances (shown in Figures 4-6 and also in Figures S2 and S3 in the Supporting Information) may be related to a better stability and quality of the dispersion (almost solution) that is strictly related to an easier access route/way toward the SPE working electrochemical area, possibly related to the creation of ordered nanochannels, where the linear diffusion processes is enhanced. In presence of random channels, the diffusion of the electroactive targets is hampered because of the presence of several additional nondiffusional paths.

\section{CONCLUSIONS}

We investigated the electrochemical performance of graphene oxide/ionic liquids screen-printed electrodes, with respect to several inorganic, organic, and biomolecules. The ionic liquids used in this study were 1-butyl-3-methylimidazolium chloride or 1-butylpyridinium chloride.

GO and ILs were synthesized and characterized, and an extensive characterization was also performed on the corresponding dispersions. GO manifests multiple $-\mathrm{OH}$ terminations which ensure an interaction with the ILs. This can be noted, for instance, in the IR spectra, especially in the case of the GO which is spontaneously deposited from BMIM$\mathrm{Cl}$, since fingerprint vibration peaks of the leftover traces of BMIM-Cl are present. The ionic liquids have a disordering effect on GO dispersion as was observed in the TE micrographs and associated SAED patterns.

In the electrochemical determinations, we observed increased peak current and significant potential shifts (especially in the case of $\beta$-NADH and catecholamines) of the modified probes in the case of both ILs and also a better and stable $I$ vs $V$ profiles. Comparison with standard CG and HOPG electrodes confirm the improved performances of GO/ILs/SPE electrodes.

Electron transfer constants were calculated for the $\mathrm{Fe}$ $(\mathrm{CN})_{6}^{3-}, \mathrm{Ru}(\mathrm{NH})_{6}^{3+}, \beta-\mathrm{NADH}$, and DOPA that all show an electrocatalytic effect. There are several factors that contribute to the improved performance of the GO/ILs modified SPEs: the large surface area, the disordering effect due to the dispersion in ionic liquids and consequent exposure of the nanoribbons to electron transfer processes, the intrinsic GO structure with oxidative groups, and unsaturated carbons also responsible for a more efficient electron transfer. Slightly better performances of Bupy-Cl over BMIM-Cl are observed, probably related to a lower $\pi-\pi$ interaction with the graphene surface. These uniformly improved performances of the GO/IL modified SPEs suggest that they may have great potential for the assembly of biosensors for various purposes.

\section{ASSOCIATED CONTENT}

\section{S Supporting Information}

Additional information as noted in text. This material is available free of charge via the Internet at http://pubs.acs.org.

\section{AUTHOR INFORMATION}

\section{Corresponding Author}

*E-mail: marilena.carbone@roma2.infn.it.

\section{Notes}

The authors declare no competing financial interest.

\section{ACKNOWLEDGMENTS}

The authors wish to thank D. R. Grosso, R. Larciprete, and R. Cimino for useful discussions. This work has been partially supported by MIUR within the Project PRIN 2008NMRHJS "DENSE".

\section{REFERENCES}

(1) Ferrari, A. C.; Meyer, J. C.; Scardaci, V.; Casiraghi, C.; Lazzeri, M.; Mauri, F.; Piscanec, S.; Jiang, D.; Novoselov, K. S.; Roth, S; Geim, A. K. Phys. Rev. Lett. 2006, 97, 187401-4.

(2) Shao, Y; Wang, J.; Wu, H.; Liu, J.; Aksay, I. A.; Lina, Y. Electroanalysis 2010, 22, 1027-1036.

(3) Dresselhaus, M. S.; Dresselhaus, G. Adv. Phys. 2002, 51, 1-186.

(4) Stankovich, S.; Piner, R.; Chen, X.; Wu, N.; Nguyen, S. T.; Ruoff, R. S. J. Mater. Chem. 2006, 16, 155-158.

(5) Berger, C.; Song, Z.; Li, T.; Li, X.; Ogbazghi, A. Y.; Feng, R.; Dai, Z.; Marchenkov, A. N.; Conrad, E. H.; First, P. N.; de Heer, W. A. J. Phys. Chem. B 2004, 108, 19912-19916.

(6) Wang, G.; Yang, J.; Park, J.; Gou, X.; Wang, B.; Liu, H.; Yao, J. J. Phys. Chem. C 2008, 112, 8192-8195.

(7) Park, S.; Ruoff, R. S. Nat. Nanotechnol. 2010, 4, 217.

(8) Cataldo, F.; Compagnini, G.; D’Urso, L.; Palleschi, G.; Valentini, F.; Angelici, G.; Braun, T. Fullerenes, Nanotubes, Carbon Nanostruct. 2010, 18, 261-272.

(9) Cataldo, F.; Compagnini, G.; Patane, G.; Ursini, O.; Angelini, G.; Ribic, P. R.; Margaritondo, G.; Cricenti, A.; Palleschi, G.; Valentini, F. Carbon 2010, 48, 2596-2602.

(10) Barone, V.; Hod, O.; Scuseria, G. E. Nano Lett. 2006, 6, 27482754.

(11) (a) Wasserscheid, P.; Welton, T. Ionic Liquids in Synthesis, 2nd ed.; Wiley-VCH: Weinheim, Germany, 2008 and refs therein. (b) Hallett, J. P.; Welton, T. Chem. Rev. 2011, 111, 3508-3576 and refs therein.

(12) (a) Tsuda, T.; Hussey, C. L. Electrochem. Soc., Interface 2007, 16, 42-49. (b) Buzzeo, M. C.; Evans, R. G.; Compton, R. C. ChemPhysChem 2004, 5, 1106-1120. 
(13) Bizzarri, C.; Conte, V.; Floris, B.; Galloni, P. J. Phys. Org. Chem. 2011, 24, 327-334 and refs therein.

(14) (a) Macfarlane, D. R.; Forsyth, M.; Howlett, P. C.; Prinlge, J. M.; Sun, J.; Aannat, G.; Neil, W.; Izgorodina, E. I. Acc. Chem. Res. 2007, 40, 1165-1173. (b) Wei, D.; Ivaska, A. Anal. Chim. Acta 2008, $607,126-135$.

(15) Valentini, F.; Romanazzo, D.; Carbone, M.; Palleschi, G. Electroanalysis 2012, 24, 711-713.

(16) Stankovich, S.; Dikin, D. A.; Piner, R. D.; Kohlhaas, K. A.; Kleinhammes, A.; Jia, Y.; Wu, Y.; Nguyen, S. B. T.; Ruoff, R. S. Carbon 2007, 45, 1558-1565.

(17) Si, Y.; Samulski, E. T. NanoLett 2008, 8 (6), 1679-1682.

(18) Park, S.; Lee, K.-S.; Bozoklu, G.; Cai, W.; Nguyen, S. B. T.; Ruoff, R. S. ACS Nano 2008, 2 (3), 572-578.

(19) Jeong, H.-K.; Lee, Y.-P.; Jin, M. H.; Kim, E. S.; Bae, J. J.; Lee, Y. H. Chem. Phys. Lett. 2009, 470, 255-258.

(20) Katsyuba, S. A.; Zvereva, E. E.; Vidiš, A.; Dyson, P. J. J. Phys. Chem. A 2007, 111, 352-370.

(21) Bard, A. J.; Faulkner, L. R. Electrochemical Methods: Fundamentals and Applications; Wiley \& Sons, Inc.: New York, 1980.

(22) Qiao, Y.; Bao, S.-J.; Li, C. M. Energy Environ. Sci. 2010, 3, 544553.

(23) Barroso, M. F.; De-Los-Santos-Alvarez, N.; Delerue-Matos, C.; Oliveira, M. B. P. P. Biosens. Bioelectron. 2011, 30, 1-12.

(24) Gorton, L.; Dominguez, E. Rev. Mol. Biotechnol. 2002, 82, 371392.

(25) Wang, Q.; Tang, H.; Xie, Q.; Tan, L.; Zhang, Y.; Li, B.; Yao, S. Electrochim. Acta 2007, 52, 6630-6637 and references therein.

(26) DuVall, S. H.; McCreery, R. L. J. Am. Chem. Soc. 2000, 122, 6759-6764 and references therein.

(27) McCreery, R. Chem. Rev. 2008, 108, 2646-2687.

(28) (a) Rochefort, A.; Wuest, J. D. Langmuir 2009, 25, 210-215. (b) Banhart, F.; Kotakoski, J.; Krasheninnikov, A. V. ACS Nano 2011, 5 (1), 26-41.

(29) Lavagnini, I.; Antiochia, R.; Magno, F. Electroanalysis 2004, 16 (6), 505-506. 